\title{
TRACE CLASS, WIDTHS AND THE FINITE APPROXIMATION PROPERTY IN BANACH SPACE
}

\author{
BY R. A. GOLDSTEIN AND R. SAEKS \\ Communicated by Robert G. Bartle, June 21, 1972
}

I. Introduction. Although there have been a number of attempts [3] to define operator classes in Banach space whose properties are analogous to the classical trace class operators of Hilbert space $[\mathbf{1}],[\mathbf{2}]$ it is generally agreed that a satisfactory definition has yet to be achieved [3]. The purpose of the present note is to introduce a new approach to the problem wherein operator widths [2], [4] in Banach space replace the eigenvalues of the Hilbert space formulation; the viability of the approach being illustrated by the formulation of a number of sufficient conditions for an operator to have the finite approximation property in terms of its widths. Moreover, unlike the previous approaches [3] the trace class operators defined via operator widths are representation independent and coincide exactly with the classical definitions in Hilbert space.

II. Definitions and results. In the sequel $X$ is a Banach space normed by $\|\cdot\|, B$ is the unit ball in $X$ and $\mathscr{L}_{n}$ is the set of $n$-dimensional subspaces of $B$. The $n$th width, $d_{n}(A)$, of an operator $A$ on $X$ is defined [4] by

$$
d_{n}(A) \equiv \inf _{L \in \mathscr{L}_{n}} \sup _{u \in B} \inf _{v \in L}\|A u-v\| .
$$

Classically, Kolmogorov [4] defined the $n$th width of a set to be a measure of the degree to which the set could be approximated by $n$-dimensional subspaces, the definition of equation (1) being that of Kolmogorov applied to $A(B)$.

Some remarks concerning the sequence $\left\{d_{n}(A)\right\}$ are as follows:

(a) $d_{0}(A)=\|A\|$;

(b) $\left\{d_{n}(A)\right\}$ is a nonincreasing sequence and $d_{n}(A) \rightarrow 0$ iff $A$ is a compact operator;

(c) (see for example [2]) for $X$ a Hilbert space and $A$ a compact linear operator on $X$, set $s_{n}(A) \equiv \lambda_{n}\left(\left(A^{*} A\right)^{1 / 2}\right) \equiv$ the $n$th eigenvalue of $\left(A^{*} A\right)^{1 / 2}$ $(n=1,2, \ldots)$ (these are called the $s$ numbers or characteristic numbers of $A)$. Then $d_{n}(A)=s_{n+1}(A)(n=0,1,2, \ldots) . A$ is an Hilbert-Schmidt or nuclear operator if the sequence $\left\{s_{n}(A)\right\}$ is an $l_{2}$ or $l_{1}$ sequence.

AMS (MOS) subject classifications (1970). Primary 47B05, 47B10, 35P05, 35P10, 46B99.

Key words and phrases. Widths, trace-class, finite approximation property, $d$-nuclear, compact operator. 
We define the "trace" classes $D_{p}$ of compact linear operators on a Banach space as

$$
D_{p} \equiv\left\{\text { A compact, linear } \mid \sum_{n=0}^{\infty} d_{n}(A)^{p}<\infty\right\} .
$$

(In the case of a Hilbert space they reduce to the classes $C_{p}$ or $S_{p}$ defined in $[\mathbf{1}]$ or $[\mathbf{2}]$.)

Define $\|\cdot\|_{p}: D_{p} \rightarrow \boldsymbol{R}$ by

$$
\|A\|_{p} \equiv\left[\sum_{0}^{\infty} d_{n}(A)^{p}\right]^{1 / p}
$$

THEOREM 1. $\|A\|_{p}$ is a norm on $D_{p}$.

An operator $A \in D_{1}$ will be called $d$-nuclear.

Before proceeding to our main theorem on the approximation of trace class operators, we introduce another sequence, $p_{n}(X)$, of positive numbers - which characterize the Banach space $X$ rather than the operator $A$ defined by

$$
p_{n}(X) \equiv \sup _{L \in \mathscr{L}_{n}} \inf _{P(L) \in P_{L}}\|P(L)\|
$$

where $\mathscr{L}_{n}$ denotes the set of all $n$-dimensional linear subspaces of $X$, and $P_{L}$ denotes the set of all projections into the subspace $L$.

(i) $1 \leqq p_{n}(X) \leqq n$.

(ii) If $X$ is a Hilbert space, $p_{n}(X) \equiv 1(\forall n)$.

(iii) $p_{n}(X)=p_{n}\left(X^{*}\right)$ if $X$ is reflective.

(iv) Murray [5] has shown that $p_{n}(X)$ grows linearly with $n$ for $X=L_{p}$ or $l_{p}(p \neq 2)$.

We say that $A$ has the finite approximation property iff $\exists$ a sequence of operators, $A_{n}$, with finite-dimensional ranges, such that $\left\|A-A_{n}\right\| \rightarrow 0$ as $n \rightarrow \infty$.

THEOREM 2. Let $A$ be a compact operator on $X$ such that

$$
\operatorname{Lim}_{n \rightarrow \infty}\left[d_{n}(A) p_{n}(X)\right]=0 \text {. }
$$

Then $A$ has the finite approximation property.

Since, in Hilbert space $p_{n}(X)=1$, and $d_{n}(A) \rightarrow 0$ for $A$ compact, we trivially obtain the classical result

COROLlaRY 3. Every compact operator on a Hilbert space has the finite approximation property. 
Although we are not able to answer the classical conjecture on the finite approximation property for any compact operator on a Banach space we can observe

Corollary 4. Every $A \in D_{1}$ has the finite approximation property.

This holds because $\left\{d_{n}(A)\right\} \in l_{1}$, hence $d_{n}(A)=O\left(1 / n^{1+\varepsilon}\right)(\varepsilon>0)$ and $d_{n}(A) p_{n}(X)=O\left(n \cdot 1 / n^{1+\varepsilon}\right)=O\left(n^{-\varepsilon}\right)(n \rightarrow \infty)$.

The standard examples of nuclear operators in Hilbert space have natural analogs in Banach space to which the corollary applies. In particular, the injection maps of Sobolev spaces $W_{0}^{s, p}$ into $L^{p}$ is $d$-nuclear, and integral operators with smooth kernels on $L^{p}$ into $L^{p}$ are $d$-nuclear.

PROOF OF THEOREM 2.

$$
\begin{aligned}
d_{n}(A) & =\min _{L \in \mathscr{L}_{n}} \max _{\|x\|=1}\|A x-L\|=\min _{L \in \mathscr{L}_{n}} \max _{\|x\|=1} \max _{x^{*} \in L^{\perp} ;\left|x^{*}\right|=1}\left\|x^{*} A x\right\| \\
& =\min _{L \in \mathscr{L}_{n}} \max _{x^{*} \in L^{\perp} ;\left|x^{*}\right|=1}\left\|A^{*} x^{*}\right\|, \quad \text { where } X^{*} \supset L^{\perp} \text { is the annihilator of } L .
\end{aligned}
$$

Lemma. $P(L)^{*}=1-P\left(L^{\perp}\right)$.

Let $\tilde{L} \in \mathscr{L}_{n}$ be the minimizing subspace for $d_{n}(A)$ (which exists since $A$ is compact). Then $\exists$ a projection $P_{n}=P(\tilde{L})$ such $\left\|P_{n}\right\|=O\left(p_{n}(X)\right)$. Set $P_{n}^{\perp} \equiv P\left(\tilde{L}^{\perp}\right)$

We then have

$$
d_{n}(A)=\sup _{x^{*} \in X^{*}} \frac{\left\|A^{*} P_{n}^{\perp} x^{*}\right\|}{\left\|P_{n}^{\perp} x^{*}\right\|} \geqq \sup _{x^{*} \in X^{*}} \frac{\left\|A^{*} P_{n}^{\perp} x^{*}\right\|}{\left\|P_{n}^{\perp}\right\|\left\|x^{*}\right\|} .
$$

Since $\left\|P_{n}^{\perp}\right\|=\left\|1-P_{n}\right\| \leqq 1+\left\|P_{n}\right\|$, we have

$$
2 p_{n}(X) d_{n}(A) \geqq \sup _{x^{*}}\left\|A^{*} P_{n}^{\perp} x^{*}\right\| /\left\|x^{*}\right\|=\left\|A^{*} P_{n}^{\perp}\right\|=\left\|\left(1-P_{n}\right) A\right\| .
$$

Setting $A_{n} \equiv P_{n} A,\left\|A-A_{n}\right\|=O\left(d_{n}(A) p_{n}(X)\right)$ and the theorem is proved.

We conjecture that the condition of Theorem 2 is also necessary.

ACKNOWLEDGEMENT. The authors wish to express their gratitude to Professor Abraham Goetz whose insights and suggestions help to guide and formulate their work.

\section{REFERENCES}

1. N. Dunford and J. T. Schwartz, Linear operators. II: Spectral theory. Selfadjoint operators in Hilbert space, Interscience, New York, 1963. MR 32 \#6181.

2. I. C. Gohberg and M. G. Krein, Introduction to the theory of linear nonselfadjoint operators in Hilbert space, "Nauka", Moscow, 1965; English transl., Transl. Math. Monographs, vol. 18, Amer. Math. Soc., Providence, R.I., 1969. MR 36 \# 3137.

3. I. M. Gel'fand and N. Ja. Vilenkin, Generalized functions. Vol. 4: Some applications of harmonic analvsis, Fizmatgiz, Moscow, 1961; English transl., Academic Press, New York, 1964. MR 26 \#4173; MR 30 \#4152. 
4. G. G. Lorentz, Approximation of functions, Holt, Rinehart and Winston, New York, 1966. MR 35 \# 4642.

5. F. J. Murray, On complementary manifolds and projections in spaces $L_{p}$ and $l_{p}$, Trans. Amer. Math. Soc. 41 (1937), 138--152.

6. L. Nirenberg, Functional analysis, Lecture Notes, New York University, 1961.

Department of Mathematics and the Department of Electrical Engineering, University of Notre Dame, Notre Dame, Indiana 46556 